\title{
Anesthetic Management of 70 Year Old Woman with Cor triatriatum Sinister
}

\author{
Yun Ho Yang, Nam Yung Kim, Sejin Kim, Minsoo Kim, Seongsik Kang* \\ Department of Anesthesiology and Pain Medicine, Institute of Medical Sciences, Kangwon National University Hospital, School of \\ Medicine, Chuncheon, South Korea \\ *Corresponding author's email: sskang [AT] kangwon.ac.kr
}

\begin{abstract}
A$ A 70 year old woman with no specific underlying disease was referred to the department of surgery of our hospital to have low anterior resection surgery for rectal cancer. Preoperative transthoracic echocardiography (TTE) revealed perforated fibromuscular septum which divides the left atrium into two chambers. Patients with Cor Triatriatum (CT) can show a clinical feature of mitral stenosis (MS) or tricuspid stenosis (TS) according to the degree of intra atrial membrane fenestration. Therefore careful anesthetic management is needed when we deal with this patients who undergo general anesthesia. Our patient was tolerable throughout the general anesthesia with propofol, volatile agent and opioid. The surgery was done without any hemodynamic event. And there was no postoperative complications.
\end{abstract}

Keywords---- (Cor triatriatum, general anesthesia, mitral stenosis, anesthetic management)

\section{INTRODUCTION}

Cor Triatriatum is a rare congenital abnormality consisting $0.1-0.4 \%$ of congenital heart disease [1]. CT results from the division of the atrium into a proximal and distal chamber. It is called cor triatriatum sinister (CTS) when it involves the left atrium and cor triatriatum dexter (CTD) when it involves the right $[2,3]$.

Generally symptomatic CT is diagnosed in childhood whereas asymptomatic CT is diagnosed in adulthood incidentally. The symptoms of CT depend on the size of the orifice between the proximal and the distal chamber. The obstruction is sufficient to create a pressure gradient when the foramen is small resembling MS or TS [2,4]. CT can be accompanied by pulmonary artery stenosis or atresia, atrial septal defect, mitral regurgitation and tricuspid valve abnormalities. Adult patient with uncorrected CT require particularly careful anesthetic management [5].

In this report, we describe case of 70 year old woman with CTS which was diagnosed incidentally in her preoperative evaluation and discuss about the anesthetic management of CTS patient.

\section{PRESENTATION OF CASE}

A 70 year old woman diagnosed rectal cancer with no specific underlying disease was referred to the department of surgery of our hospital. She was scheduled to undergo open low anterior resection. She was $144.1 \mathrm{~cm}$ tall and weighed $46.2 \mathrm{~kg}$. Preoperative routine laboratory tests, chest X-ray and ECG were normal. Her preoperative blood pressure was 130/80, heart rate 82 , respirations 15 , and room air oxygen saturation $98 \%$. She had no respiratory symptoms such as exertional dyspnea, orthopnea and hemoptysis. Transthoracic echocardiography demonstrated a membrane in the left atrium dividing it into two chambers, the proximal part and the distal part (Fig 1). It was established as class A based on Lam classification [6].

There was no flow obstruction with normal LV function: LV ejection fraction of $65 \%$ with no RWMA. RV wall thickness was not detected and the RV end-systolic pressure was $35 \mathrm{mmHg}$ suggesting absence of pulmonary hypertension. Further findings were LA enlargement and minimal mitral regurgitation. The foramen was large enough to make the intra-atrial pressure gradient be barely noticeable.

In the operating room, standard monitoring was initiated including ECG, non-invasive blood pressure, pulse oximetry and Bispectral index (BIS). During preoxygenation, the patient was given intravenous $2 \%$ lidocaine $20 \mathrm{mg}$, and propofol 80 $\mathrm{mg}$, rocuronium $40 \mathrm{mg}$. After endotracheal intubation with inner diameter size $7.0 \mathrm{~mm}$ of plain tube, anesthesia was maintained with sevoflurane and $\mathrm{O} 2$-air ( $\mathrm{FiO} 2 \mathrm{0.5}$ ). Ventilator mode was volume control (tidal volume 400ml, $\mathrm{RR} \mathrm{12/min,}$ positive end expiratory pressure $5 \mathrm{~cm} \mathrm{H} 2 \mathrm{O}$ ). 


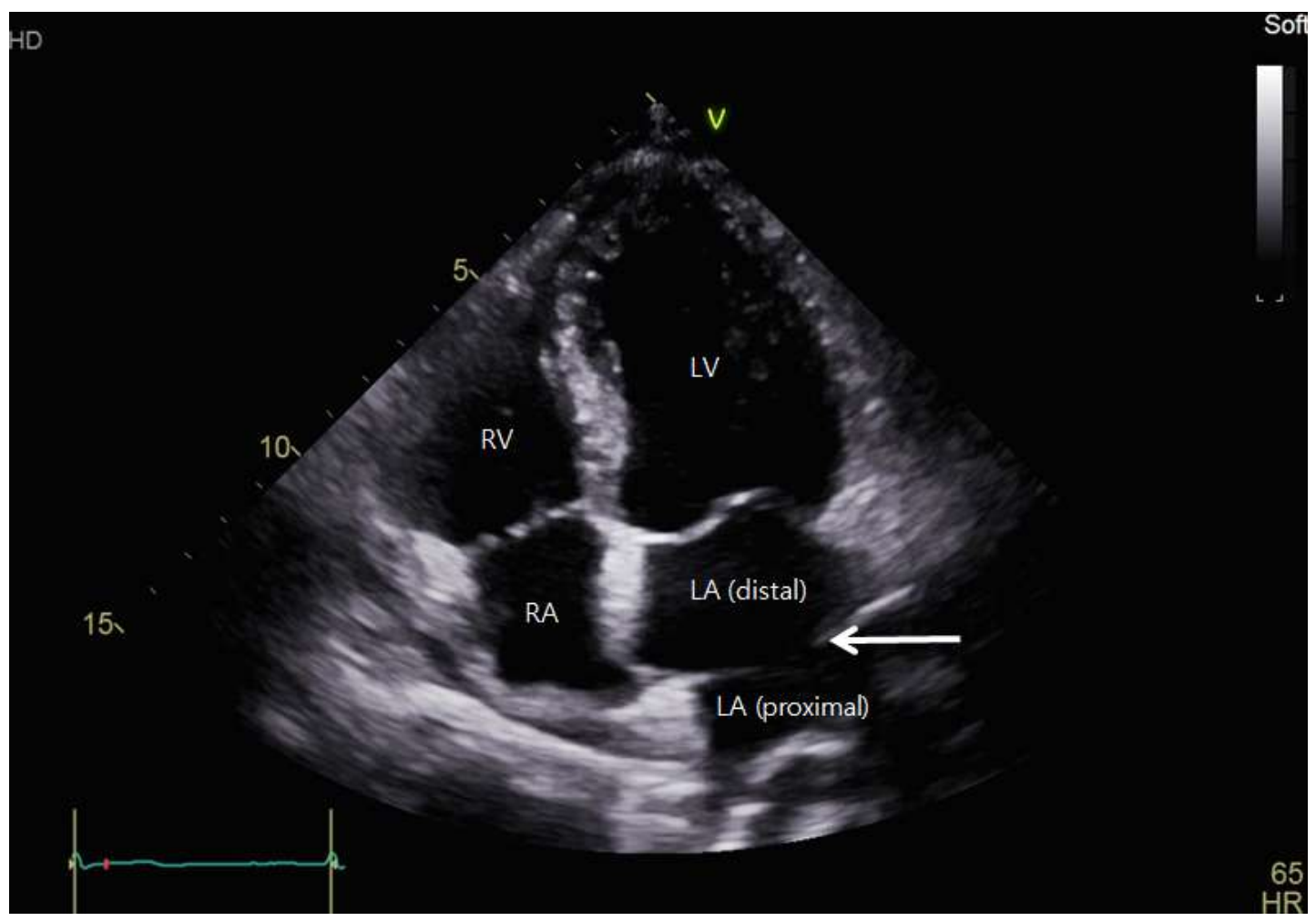

Fig. 1. Four-chamber view of transthoracic echocardiography demonstrating membrane (arrow) dividing left atrium into the proximal and distal chamber.

$R A=$ right atrium $; R V=$ right ventricle $; A=$ left atrium,$L V=$ left ventricle

Arterial cannulation was done at the right radial artery with 20 gauge. Intravenous line was done at left hand with 16 gauge. During operation, the systolic blood pressure maintained $100-140 \mathrm{~mm} \mathrm{Hg}$, heart rate $60-90 \mathrm{beats} / \mathrm{min}$, end tidal CO2 30$35 \mathrm{mmHg}$ and BIS 40-60. Fentanyl was intermittent injected for blood pressure control. Rocuronium was injected every hour at a dose of $0.25 \mathrm{mg} / \mathrm{kg}$. After operation is done, Sugammadex was given at a dose of $4 \mathrm{mg} / \mathrm{kg}$. 10 minutes later, the patient was extubated and transfer to a post-anesthesia care unit (PACU).

The surgery took 330 minutes estimating blood loss of $300 \mathrm{ml}$ and there was no complication during perioperative period. Crystalloids $(3500 \mathrm{ml})$ were infused and the total urine output was $445 \mathrm{ml}$. The vital sign maintained stable while the patient was staying in PACU and she was transferred to general ward after fully awakened from anesthesia

\section{DISCUSSION}

CT results from the division of the atrium into two chambers by a perforated fibromuscular septum. CTS is a more common condition than CTD.

Symptomatic CT is usually diagnosed in childhood and is accompanied by other congenital heart disease. These young patients get surgical treatment as soon as possible since it can be mortal if not treated [5].

Asymptomatic CT is revealed in adulthood. As the utilization of TTE is generalized in these days, more asymptomatic CT cases are detected. And this makes anesthesiologist encounter asymptomatic CT more frequently in anesthetic field. Most of asymptomatic CTS is tolerated during surgical anesthesia. However, CTS with hyperdynamic circulatory state may have symptomatic feature like mitral valve stenosis. Therefore the severity of CTS and detailed symptoms should be cautiously evaluated before surgery. During the operation, it is important to maintain left and right ventricular preloads. That means hemodynamic principles used for management of mitral stenosis should be followed maintaining euvolemia, avoiding tachycardia, hypertension and increased pulmonary vascular resistance resulting from hypoxia and hypercapnia. And maintenance of normal sinus rhythm is important in CT patient [5,7]. Also, avoiding excessive positive expiratory pressure and the Trendelenberg position can prevent an increase of RA pressure.

In this case, we made some efforts to provide balanced anesthesia: BIS was maintained 40-60 to keep adequate anesthetic depth, intermittent fentanyl was used to maintain heart rate 60-90 beat/min avoiding tachycardia.

Non-invasive tool that can accurately evaluate the morphology of the extra-membrane and the associated cardiac lesions is a TTE. Cardiac CT or MRI can be helpful for visualizing intra atrial membrane fenestration in addition. Transesophageal 
echocardiography (TEE) plays an important role in confirming the diagnosis when there is a limitations in getting image from TTE. Intraoperative assessment of cardiac contractility and volume status by TEE would be the most valuable monitoring equipment [8]. However, our patient did not undergo TEE since she had no clinical symptom and there was no flow obstruction with large membrane fenestration when examined with TTE. In this case, the surgery was well done through balanced anesthetic management without any perioperative complication.

\section{CONCLUSION}

There are increasing population of asymptomatic CT patients as the utilization of TTE is generalized. As asymptomatic patients may become symptomatic under hyperdynamic state, especial caution is needed in general anesthesia of those patients. Our non-obstructive CT patient had no perioperative complication that could be accompanied by their CT defect. Although there is rare perioperative problem in CT patient under general anesthesia, balanced anesthesia concerning hemodynamic principles should be performed by anesthesiologist to prevent development of feature like valvular stenosis

\section{REFERENCES}

1. Wolf WJ. Diagnostic features and pitfalls in the two-dimensional echocardiographic evaluation of a child with cor triatriatum. Pediatr Cardiol. 1986;6(4):211-3.

2. Nassar $\mathrm{PN}^{1}$, Hamdan RH. Cor Triatriatum Sinistrum: Classification and Imaging Modalities. Eur J Cardiovasc Med. 2011 Jan;1(3):84-7.

3. Yarrabolu TR ${ }^{1}$, Simpson L, Virani SS, Arora H, Navarijo J, Stainback RF. Cor triatriatum dexter. Tex Heart Inst J. 2007;34(3):383-5.

4. Abbasi $\mathrm{K}^{1}$, Sadeghian H, Ghafari-Marandi N, Sadeghian A. Cor triatriatum: preoperative diagnosis and successful surgical correction in a four-year-old girl. J Tehran Heart Cent. 2010 Summer;5(3):153-5. Epub 2010 Aug 31.

5. Scavonetto F, Yeoh TY, Welch TL, Weingarten TN, Sprung $\mathbf{J}^{1}$. Anesthesia and cor triatriatum. Ann Card Anaesth. 2014 Apr-Jun;17(2):111-6.

6. LAM CR, GREEN E, DRAKE E. Diagnosis and surgical correction of 2 types of triatrial heart. Surgery. 1962 Jan;51:127-37.

7. Das $\mathrm{SN}^{1}$, Kiran U, Bhan A, Sahu T. Perioperative management of cor triatriatum with congenitally corrected transposition of great arteries. Ann Card Anaesth. 2006 Jan;9(1):49-52.

8. Ikegaki $\mathbf{J}^{1}$, Kimura Y, Mikawa K, Obara H, Oka Y. Cor triatriatum in an adult patient: implications of intraoperative TEE for anesthetic management. J Cardiothorac Vasc Anesth. 1992 Apr;6(2):215-7. 